\title{
29. On Deformations of Compactifiable Complex Manifolds
}

\author{
By Yujiro Kawamata \\ Department of Mathematics, University of Tokyo
}

(Communicated by Kunihiko KodaIRA, M. J. A., Feb. 12, 1977)

In this note we shall extend the deformation theory of compact complex manifolds to compactifiable ones defined below.

1. We fix our notation.

$\bar{X}$ : a compact complex manifold,

$\bar{D}$ : a closed analytic subset of $\bar{X}$ (not necessarily reduced),

$X:=\bar{X}-\bar{D}$,

$I_{\bar{D}}:$ the ideal sheaf of $\bar{D}$ in $\mathcal{O}_{X}$,

$T_{X}(\log \bar{D})$ : the subsheaf of the tangent sheaf $T_{X}$ consisting of derivations of $\mathcal{O}_{X}$ which send $I_{\bar{D}}$ into itself.

$\bar{D}$ is said to be of simple normal crossing if (1) $\bar{D}=\bigcup_{i=1}^{h} \bar{D}_{i}$ where the $\bar{D}_{i}$ are complex submanifolds of $\bar{X}$, and (2) for each $p \in \bar{X}$, there exist a neighborhood $U$ of $p$ and a system of local coordinates $\left\{z_{1}, \cdots, z_{n}\right\}$ on $U$ such that $\bar{D}_{i}=\left\{z_{r_{i+1}}=\cdots=z_{r_{i+1}}=0\right\}$ for $1 \leqslant i \leqslant h$, where the $r_{i}$ are integers such that $-1 \leqslant i \leqslant n$ and $r_{i} \leqslant r_{j}$ if $i \leqslant j$ and we put $z_{0}=1$ by convention. In that case $\bar{X}$ is called a non-singular compactification of $X$ and $(X, \bar{X}, \bar{D})$ is called a non-singular triple. For a fixed $X$, a bimeromorphic equivalence class $\mathfrak{m}$ of non-singular compactifications of $X$ is called a meromorphic structure of $X$. A pair $(X, \mathfrak{m})$ is called a compactifiable complex manifold.

By a family of logarithmic deformations of a non-singular triple we mean a 7-tuple $\mathscr{F}=\left(\mathfrak{X}, \overline{\mathfrak{X}}, \bar{D}, \bar{\pi}, S, s_{0}, \bar{\psi}\right)$ such that (1) $\pi: \overline{\mathfrak{X}} \rightarrow S$ is a proper smooth morphism of (not necessarily reduced) complex spaces $\overline{\mathfrak{X}}$ and $S,(2) \bar{D}$ is a closed analytic subset of $\overline{\mathfrak{X}}$ and $\mathfrak{X}=\overline{\mathfrak{X}}-\bar{D},(3) \bar{\psi}: \bar{X}$ $\rightarrow \pi^{-1}\left(s_{0}\right)$ is an isomorphism such that $\bar{\psi}(X)=\pi^{-1}\left(s_{0}\right) \cap \mathfrak{X}$, and (4) $\bar{\pi}$ is locally a projection of a product space as well as the restriction of it to D. A family of compactifiable deformations of a compactifiable complex manifold $(X, \mathfrak{m})$ is a 5 -tuple $\left(\mathfrak{X}, \pi, S, s_{0}, \psi\right)$ obtained from the 7-tuple above.

Theorem 1. We have the following exact sequences:

$$
0 \longrightarrow T_{X}(-\bar{D}) \longrightarrow T_{X}(\log \bar{D}) \longrightarrow T_{\bar{D}} \longrightarrow 0
$$

where $T_{\bar{D}}$ is the sheaf of derivations $\operatorname{Der}_{\mathcal{O}_{\bar{D}}}\left(\mathcal{O}_{\bar{D}}, \mathcal{O}_{\bar{D}}\right)$.

$$
0 \longrightarrow T_{X}(\log \bar{D}) \longrightarrow T_{X} \longrightarrow N_{\bar{D}} \longrightarrow 0
$$

where $N_{\bar{D}}=\operatorname{Coker}\left(T_{\bar{D}} \rightarrow T_{X} \otimes_{\mathcal{O}_{\bar{X}}} \mathcal{O}_{\bar{D}}\right)$. 
In case where $\bar{D}$ is of simple normal crossing, we see easily $N_{\bar{D}}$ $=\bigoplus_{i=1}^{h} N_{\bar{D}_{i}}$ where the $N_{\bar{D}_{i}}$ are the normal sheaves of the $\bar{D}_{i}$ in $\bar{X}$. Theorem 1 enables us to calculate several cohomology groups.

2. In this section $(X, \bar{X}, \bar{D})$ is a non-singular triple (thus $\bar{D}$ is always assumed to be of simple normal crossing.

Theorem 2. We have a semi-universal family of logarithmic deformations of $(X, \bar{X}, \bar{D})$.

First we reduce to the case where $\bar{D}$ is a divisor by Theorem 9.1 of [3]. In that case $T_{X}(\log \bar{D})$ is a locally free sheaf on $\bar{X}$ and the harmonic integral theory is available. Therefore the proof of Theorem 2 can be carried out in an almost parallel way to [6] or [1].

An admissible center (resp. a canonical center) $C$ on $\bar{X}$ with respect to $\bar{D}$ is a closed submanifold on $\bar{X}$ of codimension at least 2 contained in $\bar{D}$ satisfying the following condition: For each $p \in C$ there exist a neighborhood $U$ of $p$ in $X$ and a closed subset $E_{U}=\bigcup_{i=1}^{k} E_{U, i}$ of simple normal crossing in $U$ such that $\bar{D} \cap U=\bigcup_{i=1}^{h} E_{U, i}(h \leqslant k)($ resp. $h=k)$ and $C \cap U=\bigcap_{i \in N} E_{U, i}$ for some $N \subset[1, k]$.

Theorem 3. Let $\bar{X}^{\prime}$ be a monoidal transform of $\bar{X}$ with an admissible center $C$ with respect to $\bar{D}$ and let $\bar{D}^{\prime}=\operatorname{red}\left(f^{-1}(\bar{D})\right)$ where $f: \bar{X}^{\prime} \rightarrow \bar{X}$ is the natural morphism. Then $\bar{D}^{\prime}$ is a closed analytic subset of simple normal crossing in $\bar{X}^{\prime}$ and

$$
R f_{*} T_{X^{\prime}}\left(\log \bar{D}^{\prime}\right)=T_{X}(\log \bar{D})(\log C),
$$

where the right hand side is the intersection of $T_{X}(\log \bar{D})$ and $T_{X}(\log C)$ in $T_{X}$. In particular if $C$ is a canonical center

$$
R f_{*} T_{X^{\prime}}\left(\log \bar{D}^{\prime}\right)=T_{X}(\log \bar{D}) \text {. }
$$

Theorem 4. Let $\left(X, \bar{X}_{1}, \bar{D}_{1}\right)$ and $\left(X, \bar{X}_{2}, \bar{D}_{2}\right)$ be two non-singular triples and $f: \bar{X}_{1} \rightarrow \bar{X}_{2}$ a morphism such that the following diagram

$$
x \nearrow_{\substack{\bar{X}_{2} \\ \bar{V}_{2}}}^{\bar{X}_{1}}
$$

is commutative. Then for an arbitrary family $\left(\mathfrak{X}, \overline{\mathfrak{X}}_{1}, \bar{D}_{1}, \bar{\pi}_{1}, S_{1}, s_{0}, \bar{\psi}_{1}\right)$ of logarithmic deformations of $\left(X, \bar{X}_{1}, \bar{D}_{1}\right)$, there exist an open neighborhood $S_{2}$ of $s_{0}$ in $S_{1}$ and a family $\left(\left.\mathfrak{X}\right|_{S_{2}}, \overline{\mathfrak{X}}_{2}, \bar{D}_{2}, \bar{\pi}_{2}, S_{2}, s_{0}, \bar{\Psi}_{2}\right)$ of logarithmic deformations of $\left(X, \bar{X}_{1}, \bar{D}_{1}\right)$ and a morphism $\mathrm{f}:\left.\overline{\mathfrak{X}}_{1}\right|_{S_{2}} \rightarrow \overline{\mathfrak{X}}_{2}$ such that $f \circ \bar{\psi}_{1}=\bar{\psi}_{2} \circ f$ on $\bar{X}_{1}$ and the following diagram

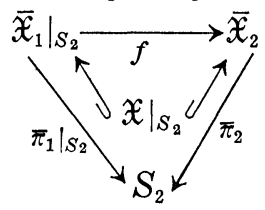


is commutative.

By Theorem 9.1 of [3], we reduce to the case where $\bar{D}_{1}$ and $\bar{D}_{2}$ are divisors. Then by Theorem 8.1 of loc. cit., we prove the theorem.

Theorem 5. With the same notation as Theorem 4, we assume furthermore that $\bar{D}_{1}$ and $\bar{D}_{2}$ are divisors and $f$ is allowable in the sense of toroidal embeddings [5]. Then the set of germs of families of compactifiable deformations of $X$ induced by the families of logarithmic deformations of $\left(X, \bar{X}_{1}, \bar{D}_{1}\right)$ coincides with that of $\left(X, \bar{X}_{2}, \bar{D}_{2}\right)$.

From Theorems 2, 3 and 4, we obtain the following four theorems.

Theorem 6. Let $\mathscr{F}=\left(\mathfrak{X}, \overline{\mathfrak{X}}, \bar{D}, \bar{\pi}, S, s_{0}, \bar{\psi}\right)$ be a family of logarithmic deformations of $(X, \bar{X}, \bar{D})$. If the Kodaira-Spencer map $\rho: T_{S, s_{0}}$ $\rightarrow H^{1}\left(\bar{X}, T_{X}(\log \bar{D})\right)$ is surjective and $S$ is regular then $\mathcal{F}$ is versal at $s_{0}$.

Theorem 7. If $H^{1}\left(\bar{X}, T_{\bar{X}}(\log \bar{D})\right)=0$, then $(X, \bar{X}, \bar{D})$ is rigid.

Theorem 8. Let $\mathscr{F}=\left(\mathfrak{X}, \bar{X}, \bar{D}, \bar{\pi}, S, s_{0}, \bar{\psi}\right)$ be a family of logarithmic deformations of $(X, \bar{X}, \bar{D})$. Assume that $\operatorname{dim} H^{1}\left(\bar{X}_{t}, T_{X_{t}}\left(\log \bar{D}_{t}\right)\right)$ is constant and $\rho_{t}: T_{S, t} \rightarrow H^{1}\left(\bar{X}_{t}, T_{X_{t}}\left(\log \bar{D}_{t}\right)\right)$ is zero for all $t \in S$, where $\bar{X}_{t}$ $=\bar{\pi}^{-1}(t)$ and $\bar{D}_{t}=\bar{D} \cap \bar{X}_{t}$. Then $\mathscr{F}_{\text {is }}$ a trivial family near $s_{0}$.

Theorem 9. If $H^{2}\left(\bar{X}, T_{X}(\log \bar{D})\right)=0$, then there exists a semiuniversal family $\left(\mathfrak{X}, \overline{\mathfrak{X}}, \bar{D}, \bar{\pi}, S, s_{0}, \bar{\psi}\right)$ of logarithmic deformations of $(X, \bar{X}, \bar{D})$ such that $S$ is regular.

By a semi-complex torus we mean a compactifiable complex manifold whose underlying complex manifold is a topologically trivial principal $\left(C^{*}\right)^{d}$-bundle over a complex torus and whose meromorphic structure is a natural one.

Theorem 10. A small compactifiable deformation of a semi-complex torus is again a semi-complex torus.

To prove the theorem we use the quasi-Albanese map extended for compactifiable complex manifolds (cf. [4]).

3. The technique developed so far is applicable to the study of equi-singular deformations of an isolated singularity. Let $X$ be a Stein neighborhood of the origin in $C^{n}$ and $D$ a reduced closed analytic subset of $X$ such that Sing $D=\{0\}$. By [2] we can find a morphism $f: \tilde{X} \rightarrow X$ by successive permissible monoidal transformations such that $\tilde{D}=\operatorname{red}\left(f^{-1}(D)\right)$ is of simple normal crossing.

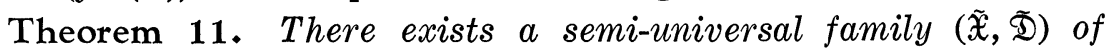
logarithmic deformations of $(\tilde{X}, \tilde{D})$. Moreover, blowing down $\mathfrak{D}$, we obtain a flat deformation (D) of $D$.

\section{References}

[1] A. Douady: Le problème des modules pour les variétés analytiques complexes. Sem. Bourbaki, $n^{\circ} 277(1965 / 65)$.

[2] H. Hironaka: Resotution of singularities of an algebraic variety over a field of characteristic zero. Ann. of Math., 79, 109-326 (1964). 
[3] E. Horikawa: On deformations of holomorphic maps. III. Math. Ann., 222, 275-282 (1976).

[4] S. Iitaka: Logarithmic forms of algebraic varieties. J. Fac. Sci. Univ. Tokyo, 23, 525-544 (1975).

[5] G. Kempf et al.: Toroidal Embeddings. I. Springer Lecture Note, 339 (1973).

[6] M. Kuranishi: Deformations of Compact Complex Manifolds. Les Press de l'Université de Montréal, Canada (1971). 